\title{
Statistics of silicate units in binary glasses
}

Anuraag Gaddam', Lionel Montagne, and José M. F. Ferreira

Citation: The Journal of Chemical Physics 145, 124505 (2016); doi: 10.1063/1.4963341

View online: http://dx.doi.org/10.1063/1.4963341

View Table of Contents: http://aip.scitation.org/toc/jcp/145/12

Published by the American Institute of Physics

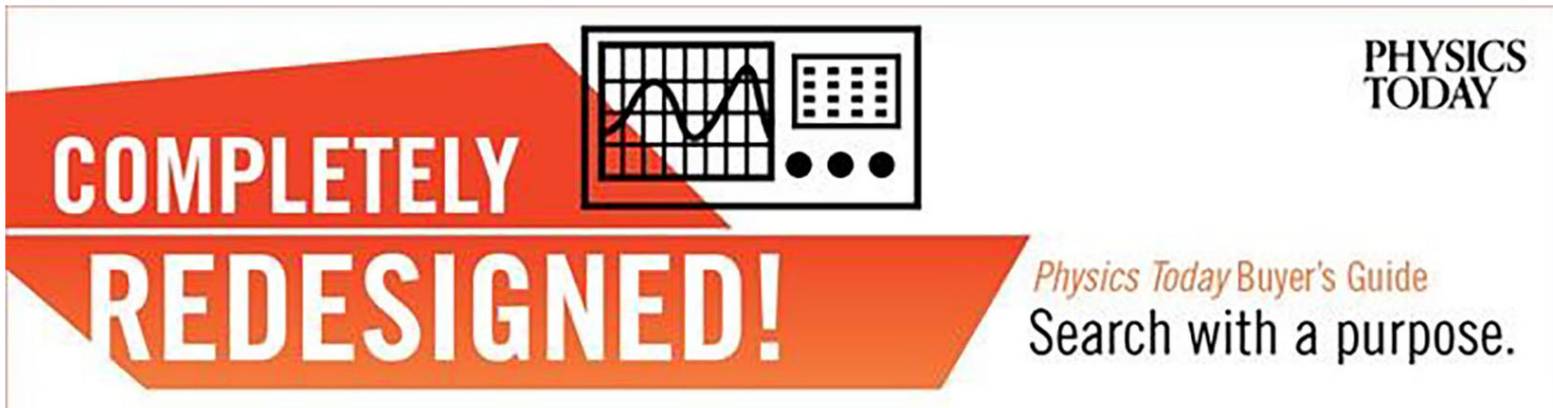




\title{
Statistics of silicate units in binary glasses
}

\author{
Anuraag Gaddam, ${ }^{1, a)}$ Lionel Montagne, ${ }^{2}$ and José M. F. Ferreira ${ }^{1}$ \\ ${ }^{1}$ Department of Materials and Ceramics Engineering, CICECO, University of Aveiro, \\ 3810-193 Aveiro, Portugal \\ ${ }^{2}$ Université Lille, CNRS, Centrale Lille, ENSCL, Université Artois, UMR 8181, UCCS-Unité de Catalyse et \\ Chimie du Solide, F-59000 Lille, France
}

(Received 20 June 2016; accepted 12 September 2016; published online 29 September 2016)

\begin{abstract}
In this paper, we derive a new model to determine the distribution of silicate units in binary glasses (or liquids). The model is based on statistical mechanics and assumes grand canonical ensemble of silicate units which exchange energy and network modifiers from the reservoir. This model complements experimental techniques, which measure short range order in glasses such as nuclear magnetic resonance $(N M R)$ spectroscopy. The model has potential in calculating the amounts of liquid-liquid phase segregation and crystal nucleation, and it can be easily extended to more complicated compositions. The structural relaxation of the glass as probed by $N M R$ spectroscopy is also reported, where the model could find its usefulness. Published by AIP Publishing. [http://dx.doi.org/10.1063/1.4963341]
\end{abstract}

\section{INTRODUCTION}

In binary alkali $\left(\mathrm{R}^{+1} ; \mathrm{R} \in\{\mathrm{Li}, \mathrm{Na}, \mathrm{K}, \mathrm{Rb}, \mathrm{Cs}\}\right)$ or alkaline earth $\left(\mathrm{R}^{+2} ; \mathrm{R} \in\{\mathrm{Mg}, \mathrm{Ca}, \mathrm{Sr}, \mathrm{Ba}\}\right)$ silicate glasses (or liquids), silicates form tetrahedral structures that are connected to each other by corner sharing. ${ }^{1}$ The oxygens in these glasses exist in three forms, namely, (1) free oxygens $\left(\mathrm{FOs}, \mathrm{O}^{-2}\right)$, (2) non-bridging oxygens $\left(\mathrm{NBO}\right.$ s, $\left.\mathrm{O}^{-1}\right)$, and (3) bridging oxygens $\left(\mathrm{BOs}, \mathrm{O}^{0}\right)$. Though, at lower concentrations of $\mathrm{R}_{2} \mathrm{O}$ (or $\mathrm{RO}$ ), the amount of $F O \mathrm{~s}$ in the composition is negligible., 2,3 Providentially, these compositions are of interest to the glass science because of their glass forming ability. The $B O \mathrm{~s}$ and $N B O \mathrm{~s}$ are present on the corners of silicate tetrahedra where the $B O$ s act as connectors between two tetrahedra, while the $N B O$ s terminate the connectivity of a given tetrahedron. Therefore, depending upon the number of $N B O s$ and $B O s$ on a given silicate tetrahedron, the tetrahedron can be classified by $Q_{n}$ notation where $n \in\{[0,4] \cap \mathbb{N}\}$ is the number of $B O$ s on a given silicate tetrahedron.

Studies on the distribution of $Q_{n}$ units are ubiquitous in the field of silicate based glasses. Techniques such as nuclear magnetic resonance (NMR) and Raman spectroscopies are routinely employed to assess the distribution of structural units. Also, there are many mathematical models that theoretically address this issue to gain fundamental understanding of this distribution. The binary model presumes only two types of $Q_{n}$ units at each composition without taking account of the speciation reaction (R1); therefore, it only describes the distribution that corresponds only to crystalline silicates but not glasses. A pure statistical model based on binomial distribution was suggested, supposing a completely random distribution of $\mathrm{BOs}$ and $\mathrm{NBOs}{ }^{4}$ However, this model does not take into account the temperature effects. Further, Brandriss et $a l^{5}$ suggested

\footnotetext{
a) Author to whom correspondence should be addressed. Electronic mail: anuraagg@ua.pt. Tel.: +351 234370242.
}

a thermodynamic model to take temperature effects into consideration. In this model, equilibrium constants $\left(k_{n}\right)$ are experimentally measured by assuming a speciation reaction (R1) and using the van't Hoff equation $\Delta H_{n}$ is calculated as follows:

$$
\begin{gathered}
2 Q_{n} \leftrightarrow Q_{n-1}+Q_{n+1} \quad \Delta H_{n}, \\
k_{n}(T)=\frac{\left[Q_{n+1}\right]\left[Q_{n-1}\right]}{\left[Q_{n}\right]^{2}} \Gamma, \\
\frac{\partial \ln k_{n}(T)}{\partial T}=\frac{\Delta H_{n}}{R T^{2}}, \\
\frac{\Delta H_{n}}{R}=\frac{\ln k_{n}\left(T_{2}\right)-\ln k_{n}\left(T_{1}\right)}{\left(\frac{1}{T_{1}}-\frac{1}{T_{2}}\right)},
\end{gathered}
$$

where $\Gamma \approx 1$ corresponds to a function of activity coefficients. By measuring $k_{n}$ at any two different temperatures by $N M R$ or Raman spectroscopy, $\Delta H_{n}$ is evaluated, and using the value of $\Delta H_{n}, k_{n}$ at other temperatures could be calculated. Another thermodynamic model of associated solutions was proposed, which employs rigorous thermodynamic theory of affinity. ${ }^{6-9}$ This model uses Gibbs free energy of formation for all the crystalline compounds formed in a particular glass system. Nevertheless, all these models use either pure statistics or macroscopic thermodynamics and therefore have their own limitations. A statistical mechanical model was proposed by Mauro ${ }^{10}$ for the glass systems having a single network modifier and multiple network formers. This model is based on non-central hypergeometric distribution where the bias is weighted by Boltzmann factors. The model provides a mathematical description for the distribution network modifiers among various network formers; however, it does not address the problem of $Q_{n}$ distribution.

Therefore, in this paper we introduce a new statistical mechanical model for binary silicate glass systems in order to address the problem of $Q_{n}$ distribution from a fundamental standpoint. The model assumes presence of 
no FOs. The model has a huge technological importance and has a potential to deal with some of the open problems in the field of glass science such as liquid-liquid phase segregation (LLPS), crystal nucleation, and structural relaxation.

\section{FORMULATION OF THE MODEL}

\section{A. Defining silicate units}

As described in the Introduction, silicate units are defined by the $Q_{n}$ notation based on the number of $B O(\mathrm{~s})$ that surround a given $\mathrm{Si}$ atom. However, there have been a number of suggestions from $N M R$ spectroscopy that in glass compositions, silicate units can be further described by considering the next-nearest neighbors. ${ }^{11-13}$ Based on this new description, the units can be defined as $Q_{4}{ }^{i j k l}(35), Q_{3}{ }^{i j k}(20)$, $Q_{2}{ }^{i j}$ (10), $Q_{1}{ }^{i}(4)$, and $Q_{0}(1)$, where $i, j, k, l \in\{[1,4] \cap \mathbb{N}\}$. For example, a $Q_{3}{ }^{334}$ unit would have three $B O \mathrm{~s}$, out of which, two are connected to $Q_{3}$ units and one is connected to a $Q_{4}$ unit (Fig. 1). According to this new definition, there would be 70 different types of silicate units, from all the combinations of the superscripts as listed in Table I. Howbeit, in this paper we introduce a new $S_{n}{ }^{m}$ notation that is more suitable for the derivation of the model where $n \in\{[0,4] \cap \mathbb{N}\}$, while $m \in\{[1, m(n)] \cap \mathbb{N}\}$. Here $n$ has same meaning as in $Q$ notation, corresponding to the internal structure of the unit, i.e., the amount of alkali or alkaline metal ions present in it. While $m$ corresponds to the external structure, i.e., the types of units a given silicate unit is connected to, and $m$ maps a particular combination of $i j k l$ of a $Q$ notation. A comparison between $Q$ notation and $S$ notation is shown in Table I. In this paper, both notations are used interchangeably according to the convenience (Fig. 1). We also define different types of $B O \mathrm{~s}$ in the glass by $O_{i j}$ notation,

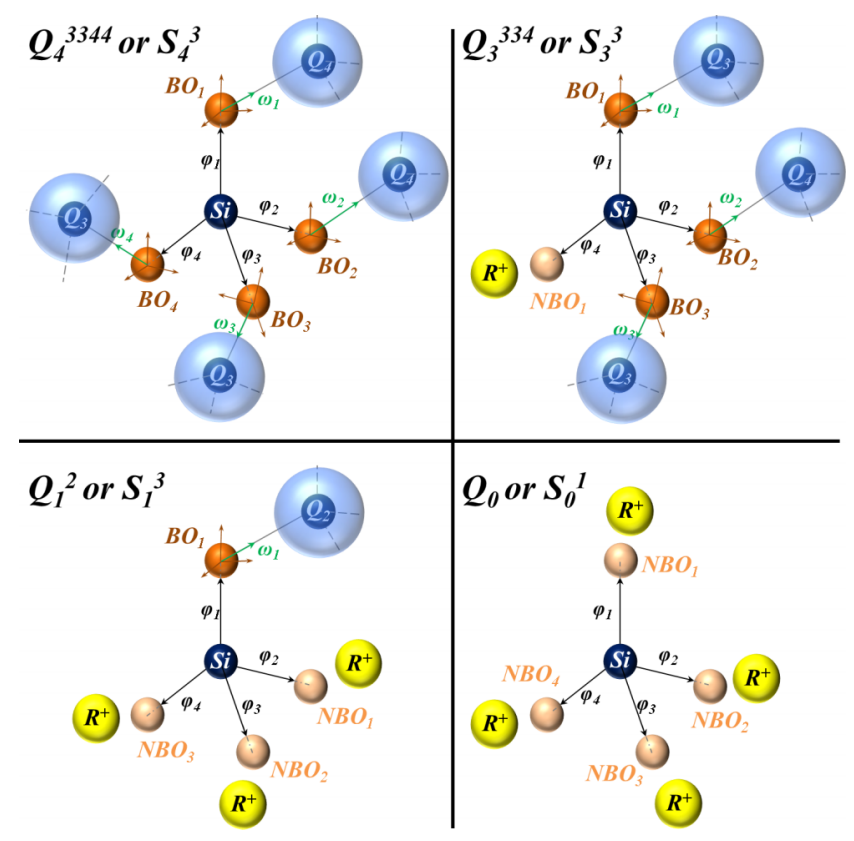

FIG. 1. Examples of silicate units and basis vectors corresponding to $\boldsymbol{\Phi}$ and $\boldsymbol{\Omega}$. where $O_{i j}$ is a $B O$ connecting $Q_{i}$ and $Q_{j}(i, j \in\{[1,4] \cap \mathbb{N}\})$ units together.

\section{B. Statistical treatment}

Consider a liquid of either alkali $\left(\mathrm{R}^{+1}\right)$ or alkaline earth $\left(\hat{\mathrm{R}}^{+2}\right)$ silicate composition given by

$$
\begin{gathered}
\mathrm{R}_{2} \mathrm{O} \text { or ŔO }: x, \\
\mathrm{SiO}_{2}: 1 .
\end{gathered}
$$

Here, the amount of $\mathrm{SiO}_{2}$ is scaled to unity and the addition of the network modifiers is given by the variable $x$, where $x \in[0,2]$, which corresponds to $\mathrm{R}_{2} \mathrm{O} \% \in[0,2 / 3]$. If $P_{n}{ }^{m}$ is probability (or fraction) of occurrence of a $S_{n}{ }^{m}$ microstate, then the constraints (1)-(3) must hold, which are constraints corresponding to the amounts of $\mathrm{SiO}_{2}$, energy, and $\mathrm{R}_{2} \mathrm{O}$ or ŔO, respectively,

$$
\begin{gathered}
\sum_{n, m} P_{n}^{m}=1, \\
\sum_{n, m} E_{n}^{m} P_{n}^{m}=\langle E\rangle, \\
\sum_{n, m} n P_{n}^{m}=2[2-\langle x\rangle]=\left\langle N_{B O}\right\rangle,
\end{gathered}
$$

where $E_{n}{ }^{m}$ is the energy of a given $S_{n}{ }^{m}$ microstate, while $\langle E\rangle,\langle x\rangle$ and $\left\langle N_{B O}\right\rangle \in[0,4]$ are the expected values of energy, composition, and the amount of $\mathrm{BO}$ s for a given ensemble. Additionally, because $S_{n}{ }^{m}$ notation takes into consideration the network linkages with its neighbors, there would be 10 more additional internal constraints connecting the probabilities of different $S_{n}{ }^{m}$ microstates corresponding to the 10 different types of $B O \mathrm{~s}\left(O_{i j}\right)$. The equations are presented in Subsection 1 of the Appendix and they take the form given by the following:

$$
\sum_{n, m}(i, j)_{n}^{m} P_{n}^{m}=0 .
$$

The coefficients $(i, j)_{n}{ }^{m}$ represent the number of network connections between $Q_{i}$ and $Q_{j}$ silicate units originating from a given $S_{n}{ }^{m}$ unit. The following examples illustrate the physical meaning of these coefficients:

- The value of $(3,2)_{3}{ }^{8}$ which corresponds to the microstate $S_{3}{ }^{8}$ (or $Q_{3}{ }^{224}$ ) would be 2 because there are two $3 \rightarrow 2$ connections.

- The value of $(4,3)_{3}{ }^{1}$ which corresponds to the microstate $S_{3}{ }^{1}$ (or $Q_{3}{ }^{444}$ ) would be -3 because there are three $3 \rightarrow 4$ connections, and the negative sign implies the reversal of the originating direction.

- The value of $(4,3)_{3}{ }^{20}$ which corresponds to the microstate $S_{3}{ }^{20}$ (or $Q_{3}{ }^{111}$ ) would be 0 because of the non-existence of any $4 \rightarrow 3$ connections.

All the values of the coefficients $(i, j)_{n}{ }^{m}$ are presented in Table I. Basically, Equations (3) and (4) represent constraints corresponding to chemical composition and network connectivity, respectively. The entropy generated by 
TABLE I. Comparison between $Q$ and $S$ notations, and constants associated to network connectivity.

\begin{tabular}{|c|c|c|c|c|c|c|c|c|}
\hline No. & $S_{n}{ }^{m}$ & $Q_{n}^{i j \ldots}$ & $(4,3)_{n}{ }^{m}$ & $(4,2)_{n}{ }^{m}$ & $(4,1)_{n}{ }^{m}$ & $(3,2)_{n}{ }^{m}$ & $(3,1)_{n}{ }^{m}$ & $(2,1)_{n}^{m}$ \\
\hline \multicolumn{9}{|c|}{ Units of $Q_{4}^{i j k l}$} \\
\hline 1 & $S_{4}{ }^{1}$ & $Q_{4}{ }^{4444}$ & 0 & 0 & 0 & 0 & 0 & 0 \\
\hline 2 & $S_{4}^{2}$ & $Q_{4}{ }^{3444}$ & 1 & 0 & 0 & 0 & 0 & 0 \\
\hline 3 & $S_{4}{ }^{3}$ & $Q_{4}{ }^{3344}$ & 2 & 0 & 0 & 0 & 0 & 0 \\
\hline 4 & $S_{4}{ }^{4}$ & $Q_{4}^{3334}$ & 3 & 0 & 0 & 0 & 0 & 0 \\
\hline 5 & $S_{4}{ }^{5}$ & $Q_{4}{ }^{3333}$ & 4 & 0 & 0 & 0 & 0 & 0 \\
\hline 6 & $S_{4}{ }^{6}$ & $Q_{4}^{2444}$ & 0 & 1 & 0 & 0 & 0 & 0 \\
\hline 7 & $S_{4}{ }^{7}$ & $Q_{4}{ }^{2344}$ & 1 & 1 & 0 & 0 & 0 & 0 \\
\hline 8 & $S_{4}{ }^{8}$ & $Q_{4}{ }^{2334}$ & 2 & 1 & 0 & 0 & 0 & 0 \\
\hline 9 & $S_{4}{ }^{9}$ & $Q_{4}{ }^{2333}$ & 3 & 1 & 0 & 0 & 0 & 0 \\
\hline 10 & $S_{4}{ }^{10}$ & $Q_{4}{ }^{2244}$ & 0 & 2 & 0 & 0 & 0 & 0 \\
\hline 11 & $S_{4}{ }^{11}$ & $Q_{4}^{2234}$ & 1 & 2 & 0 & 0 & 0 & 0 \\
\hline 12 & $S_{4}{ }^{12}$ & $Q_{4}{ }^{2233}$ & 2 & 2 & 0 & 0 & 0 & 0 \\
\hline 13 & $S_{4}{ }^{13}$ & $Q_{4}^{2224}$ & 0 & 3 & 0 & 0 & 0 & 0 \\
\hline 14 & $S_{4}{ }^{14}$ & $Q_{4}{ }^{2223}$ & 1 & 3 & 0 & 0 & 0 & 0 \\
\hline 15 & $S_{4}{ }^{15}$ & $Q_{4}{ }^{2222}$ & 0 & 4 & 1 & 0 & 0 & 0 \\
\hline 16 & $S_{4}{ }^{16}$ & $Q_{4}{ }^{1444}$ & 0 & 0 & 1 & 0 & 0 & 0 \\
\hline 17 & $S_{4}{ }^{17}$ & $Q_{4}{ }^{1344}$ & 1 & 0 & 1 & 0 & 0 & 0 \\
\hline 18 & $S_{4}{ }^{18}$ & $Q_{4}{ }^{1334}$ & 2 & 0 & 1 & 0 & 0 & 0 \\
\hline 19 & $S_{4}{ }^{19}$ & $Q_{4}{ }^{1333}$ & 3 & 0 & 1 & 0 & 0 & 0 \\
\hline 20 & $S_{4}{ }^{20}$ & $Q_{4}{ }^{1244}$ & 0 & 1 & 1 & 0 & 0 & 0 \\
\hline 21 & $S_{4}{ }^{21}$ & $Q_{4}{ }^{1234}$ & 1 & 1 & 1 & 0 & 0 & 0 \\
\hline 22 & $S_{4}^{22}$ & $Q_{4}{ }^{1233}$ & 2 & 1 & 1 & 0 & 0 & 0 \\
\hline 23 & $S_{4}{ }^{23}$ & $Q_{4}{ }^{1224}$ & 0 & 2 & 1 & 0 & 0 & 0 \\
\hline 24 & $S_{4}{ }^{24}$ & $Q_{4}{ }^{1223}$ & 1 & 2 & 1 & 0 & 0 & 0 \\
\hline 25 & $S_{4}{ }^{25}$ & $Q_{4}{ }^{1222}$ & 0 & 3 & 1 & 0 & 0 & 0 \\
\hline 26 & $S_{4}{ }^{26}$ & $Q_{4}{ }^{1144}$ & 0 & 0 & 2 & 0 & 0 & 0 \\
\hline 27 & $S_{4}{ }^{27}$ & $Q_{4}{ }^{1134}$ & 1 & 0 & 2 & 0 & 0 & 0 \\
\hline 28 & $S_{4}{ }^{28}$ & $Q_{4}{ }^{1133}$ & 2 & 0 & 2 & 0 & 0 & 0 \\
\hline 29 & $S_{4}{ }^{29}$ & $Q_{4}{ }^{1124}$ & 0 & 1 & 2 & 0 & 0 & 0 \\
\hline 30 & $S_{4}{ }^{30}$ & $Q_{4}{ }^{1123}$ & 1 & 1 & 2 & 0 & 0 & 0 \\
\hline 31 & $S_{4}{ }^{31}$ & $Q_{4}{ }^{1122}$ & 0 & 2 & 2 & 0 & 0 & 0 \\
\hline 32 & $S_{4}{ }^{32}$ & $Q_{4}{ }^{1114}$ & 0 & 0 & 3 & 0 & 0 & 0 \\
\hline 33 & $S_{4}{ }^{33}$ & $Q_{4}{ }^{1113}$ & 1 & 0 & 3 & 0 & 0 & 0 \\
\hline 34 & $S_{4}{ }^{34}$ & $Q_{4}{ }^{1112}$ & 0 & 1 & 3 & 0 & 0 & 0 \\
\hline 35 & $S_{4}{ }^{35}$ & $Q_{4}{ }^{1111}$ & 0 & 0 & 4 & 0 & 0 & 0 \\
\hline \multicolumn{9}{|c|}{ Units of $Q_{3}{ }^{i j k}$} \\
\hline 36 & $S_{3}{ }^{1}$ & $Q_{3}^{444}$ & -3 & 0 & 0 & 0 & 0 & 0 \\
\hline 37 & $S_{3}^{2}$ & $Q_{3}^{344}$ & -2 & 0 & 0 & 0 & 0 & 0 \\
\hline 38 & $S_{3}{ }^{3}$ & $Q_{3}^{334}$ & -1 & 0 & 0 & 0 & 0 & 0 \\
\hline 39 & $S_{3}{ }^{4}$ & $Q_{3}^{333}$ & 0 & 0 & 0 & 0 & 0 & 0 \\
\hline 40 & $S_{3}{ }^{5}$ & $Q_{3}^{244}$ & -2 & 0 & 0 & 1 & 0 & 0 \\
\hline 41 & $S_{3}{ }^{6}$ & $Q_{3}^{234}$ & -1 & 0 & 0 & 1 & 0 & 0 \\
\hline 42 & $S_{3}{ }^{7}$ & $Q_{3}^{233}$ & 0 & 0 & 0 & 1 & 0 & 0 \\
\hline 43 & $S_{3}{ }^{8}$ & $Q_{3}^{224}$ & -1 & 0 & 0 & 2 & 0 & 0 \\
\hline 44 & $S_{3}{ }^{9}$ & $Q_{3}^{223}$ & 0 & 0 & 0 & 2 & 0 & 0 \\
\hline 45 & $S_{3}{ }^{10}$ & $Q_{3}^{222}$ & 0 & 0 & 0 & 3 & 0 & 0 \\
\hline 46 & $S_{3}{ }^{11}$ & $Q_{3}^{144}$ & -2 & 0 & 0 & 0 & 1 & 0 \\
\hline 47 & $S_{3}{ }^{12}$ & $Q_{3}^{134}$ & -1 & 0 & 0 & 0 & 1 & 0 \\
\hline 48 & $S_{3}{ }^{13}$ & $Q_{3}^{133}$ & 0 & 0 & 0 & 0 & 1 & 0 \\
\hline 49 & $S_{3}{ }^{14}$ & $Q_{3}^{124}$ & -1 & 0 & 0 & 1 & 1 & 0 \\
\hline 50 & $S_{3}{ }^{15}$ & $Q_{3}^{123}$ & 0 & 0 & 0 & 1 & 1 & 0 \\
\hline 51 & $S_{3}{ }^{16}$ & $Q_{3}^{122}$ & 0 & 0 & 0 & 2 & 1 & 0 \\
\hline 52 & $S_{3}{ }^{17}$ & $Q_{3}^{114}$ & -1 & 0 & 0 & 0 & 2 & 0 \\
\hline 53 & $S_{3}{ }^{18}$ & $Q_{3}^{113}$ & 0 & 0 & 0 & 0 & 2 & 0 \\
\hline 54 & $S_{3}{ }^{19}$ & $Q_{3}^{112}$ & 0 & 0 & 0 & 1 & 2 & 0 \\
\hline 55 & $S_{3}^{20}$ & $Q_{3}^{111}$ & 0 & 0 & 0 & 0 & 3 & 0 \\
\hline
\end{tabular}


TABLE I. (Continued.)

\begin{tabular}{|c|c|c|c|c|c|c|c|c|}
\hline No. & $S_{n}{ }^{m}$ & $Q_{n}^{i j \ldots}$ & $(4,3)_{n}{ }^{m}$ & $(4,2)_{n}{ }^{m}$ & $(4,1)_{n}{ }^{m}$ & $(3,2)_{n}{ }^{m}$ & $(3,1)_{n}{ }^{m}$ & $(2,1)_{n}{ }^{m}$ \\
\hline \multicolumn{9}{|c|}{ Units of $Q_{2}^{i j}$} \\
\hline 56 & $S_{2}{ }^{1}$ & $Q_{2}{ }^{44}$ & 0 & -2 & 0 & 0 & 0 & 0 \\
\hline 57 & $S_{2}^{2}$ & $Q_{2}^{34}$ & 0 & -1 & 0 & -1 & 0 & 0 \\
\hline 58 & $S_{2}{ }^{3}$ & $Q_{2}{ }^{33}$ & 0 & 0 & 0 & -2 & 0 & 0 \\
\hline 59 & $S_{2}{ }^{4}$ & $Q_{2}^{24}$ & 0 & -1 & 0 & 0 & 0 & 0 \\
\hline 60 & $S_{2}{ }^{5}$ & $Q_{2}{ }^{23}$ & 0 & 0 & 0 & -1 & 0 & 0 \\
\hline 61 & $S_{2}{ }^{6}$ & $Q_{2}^{22}$ & 0 & 0 & 0 & 0 & 0 & 0 \\
\hline 62 & $S_{2}{ }^{7}$ & $Q_{2}{ }^{14}$ & 0 & -1 & 0 & 0 & 0 & 1 \\
\hline 63 & $S_{2}{ }^{8}$ & $Q_{2}^{13}$ & 0 & 0 & 0 & -1 & 0 & 1 \\
\hline 64 & $S_{2}{ }^{9}$ & $Q_{2}{ }^{12}$ & 0 & 0 & 0 & 0 & 0 & 1 \\
\hline 65 & $S_{2}{ }^{10}$ & $Q_{2}{ }^{11}$ & 0 & 0 & 0 & 0 & 0 & 2 \\
\hline \multicolumn{9}{|c|}{ Units of $Q_{1}{ }^{i}$} \\
\hline 66 & $S_{1}{ }^{1}$ & $Q_{1}^{4}$ & 0 & 0 & -1 & 0 & 0 & 0 \\
\hline 67 & $S_{1}{ }^{2}$ & $Q_{1}^{3}$ & 0 & 0 & 0 & 0 & -1 & 0 \\
\hline 68 & $S_{1}{ }^{3}$ & $Q_{1}^{2}$ & 0 & 0 & 0 & 0 & 0 & 1 \\
\hline 69 & $S_{1}^{4}$ & $Q_{1}^{1}$ & 0 & 0 & 0 & 0 & 0 & 0 \\
\hline \multicolumn{9}{|c|}{ Units of $Q_{0}$} \\
\hline 70 & $S_{0}{ }^{1}$ & $Q_{0}$ & 0 & 0 & 0 & 0 & 0 & 0 \\
\hline
\end{tabular}

a given distribution of $S_{n}{ }^{m}$ microstates is given by

$$
S=-k_{B} \sum_{n, m}\left[P_{n}^{m} \ln P_{n}^{m}\right],
$$

where $k_{B}$ is the Boltzmann constant. Maximizing Eq. (5) by subjecting to the constraints Eqs. (1)-(4) using the method of Lagrange multipliers would yield (Subsection 2 of the Appendix),

$$
P_{n}^{m}=\frac{1}{Z_{g r}} e^{\frac{\sum_{i \geq j}(i, j)_{n}^{m} \mu_{i j}+n \mu-E_{n}^{m}}{k_{B} T}},
$$

where $\mu$ and $\mu_{i j}$ are the chemical potentials associated to the exchange of network modifiers $\left(\mathrm{R}^{+}\right.$or $\mathrm{R}^{2+}$ ) and network connections, respectively, $T$ is the temperature, and $Z_{g} r$ is the grand canonical partition function given by

$$
Z_{g r}=\sum_{n, m} e^{\frac{\sum_{i \geq j}(i, j)_{n}^{m} \mu_{i j}+n \mu-E_{n}^{m}}{k_{B} T}} .
$$

\section{Energy consideration and quantization}

The energy associated with a given $S_{n}{ }^{m}$ microstate would be vibrational energy. ${ }^{14}$ The frequencies of the vibrational normal modes associated to a particular $S_{n}{ }^{m}$ microstate could be obtained by appropriately choosing the interatomic potentials derived from quantum mechanical calculations and then solving the characteristic equation. If each $S_{n}{ }^{m}$ microstate has $N_{n}{ }^{m}$ number of normal modes associated to it, labelled by $v \in\left\{\left[1, N_{n}{ }^{m}\right] \cap \mathbb{N}\right\}$, then a given $S_{n}{ }^{m}$ unit can be considered to be an $N_{n}{ }^{m}$ dimensional quantum harmonic oscillator. Consequently, we can represent the vibrational state of the $S_{n}{ }^{m}$ unit existing in some stationary state by a state vector $\left|S_{n}^{m}\left(\boldsymbol{k}_{\boldsymbol{n}}^{\boldsymbol{m}}\right)\right\rangle$ where $\boldsymbol{k}_{\boldsymbol{n}}{ }^{\boldsymbol{m}}$ is vector $\in \mathbb{Z}^{N_{n}^{m}}$ in positive orthant subspace, the meaning of which would be apparent subsequently. When the Hamiltonian $(\hat{H})$ acts on the state vector $\left|S_{n}^{m}\left(\boldsymbol{k}_{n}^{m}\right)\right\rangle$, it would yield

$$
\left.\hat{H}\left|S_{n}^{m}(\boldsymbol{k})=\left[\sum_{v=1}^{N_{n}^{m}}\left(\frac{1}{2}+k_{n}^{m}(v)\right) \hbar \omega_{n}^{m}(v)\right]\right| S_{n}^{m}\left(\boldsymbol{k}_{\boldsymbol{n}}^{\boldsymbol{m}}\right)\right\rangle,
$$

where $\hbar$ is the Dirac constant, ${k_{n}}^{m}(v) \in \mathbb{N}$ and $\omega_{n}{ }^{m}(v)$ are the quantum numbers and the angular frequency associated to the $v$ th mode of the quantum harmonic oscillator. Here, the vector $\boldsymbol{k}_{\boldsymbol{n}}{ }^{\boldsymbol{m}}$ corresponds to a set of quantum numbers associated to all the normal modes $\left(k(1), k(2), \ldots, k\left(N_{n}{ }^{m}\right)\right)$. In the quantum mechanical framework, the statistical probability is given by the density operator $(\hat{\rho})$, which is based on Eq. (6) and would take the form

$$
\hat{\rho}=\frac{1}{Z_{g r}} e^{\frac{\sum_{I \geq J} \widehat{I J} \mu_{i j}+\hat{n} \mu-\hat{H}}{k_{B} T}} .
$$

Here, two new operators $\widehat{I J}$ and $\hat{n}$ are introduced; they act on the state vector $\left|S_{n}^{m}\right\rangle$ and give Eigen values $(i, j)_{n}{ }^{m}$ and $n$, respectively. Both, $\widehat{I J}$ and $\hat{n}$ operators commutate with the Hamiltonian. Further, the partition function $Z_{g r}$ is given by

$$
Z_{g r}=\operatorname{Tr}\left(e^{\frac{\sum_{I \geq J} \widehat{I J} \mu_{i j}+\hat{n} \mu-\hat{H}}{k_{B} T}}\right) .
$$

When $\hat{\rho}$ acts on the state vector $\left|S_{n}^{m}\right\rangle$, it gives the probability $P_{n}{ }^{m}$,

$$
\begin{aligned}
\hat{\rho}\left|S_{n}^{m}(\boldsymbol{k})\right\rangle & =\frac{1}{Z_{g r}} e^{\frac{\sum_{I \geq J \widehat{J J} \mu_{i j}+\hat{n} \mu-\hat{H}}}{k_{B} T}}\left|S_{n}^{m}\left(\boldsymbol{k}_{n}^{m}\right)\right\rangle \\
& =\frac{1}{Z_{g r}} e^{\frac{\sum_{i \geq j}(i, j)_{n}^{m} \mu+n \mu-\sum_{v=1}^{N_{n}}\left(\frac{1}{2}+k_{n}^{m}(v)\right) \hbar \omega_{n}^{m}(\nu)}{k_{B} T}}\left|S_{n}^{m}\left(\boldsymbol{k}_{n}^{m}\right)\right\rangle .
\end{aligned}
$$


The partition function can be evaluated as

$$
\begin{aligned}
Z_{g r} & =\sum_{n, m} \sum_{k_{n}^{m}} e^{\frac{\sum_{i \geq j}(i, j)_{n}^{m} \mu_{i j}+n \mu-\sum_{v=1}^{N_{n}^{m}}\left(\frac{1}{2}+k_{n}^{m}(v)\right) \hbar \omega_{n}^{m}(v)}{k_{B} T}} \\
& =\sum_{n, m} \sum_{k_{n}^{m}} e^{\frac{\sum_{i \geq j}(i, j)_{n}^{m} \mu_{i j}+n \mu}{k_{B} T}} e^{\frac{-\sum_{v=1}^{N_{n}^{m}\left(\frac{1}{2}+k_{n}^{m}(v)\right) \hbar \omega_{n}^{m}(v)}}{k_{B} T}} \\
& =\sum_{n, m} e^{\frac{\sum_{i \geq j}(i, j)_{n}^{m} \mu_{i j}+n \mu}{k_{B} T}} \prod_{v=1}^{N_{n}^{m}}\left(\frac{1}{2 \sinh \left(\frac{\hbar \omega_{n}^{m}(v)}{2 k_{B} T}\right)}\right) \\
& =\sum_{n, m} e^{\frac{\sum_{i \geq j}(i, j)_{n}^{m} \mu_{i j}+n \mu}{k_{B} T}} Z_{n}^{m}
\end{aligned}
$$

where $Z_{n}{ }^{m}$ is the canonical partition function associated to a given $S_{n}{ }^{m}$ microstate. It can also be written in terms of Helmholtz free energy $\left(F_{n}{ }^{m}\right)$ of the quantum harmonic oscillator as

$$
Z_{n}^{m}=e^{-\frac{F_{n}^{m}}{k_{B}}}
$$

Therefore, the probability distribution of $S_{n}{ }^{m}$ microstates is given by

$$
P_{n}^{m}=\frac{1}{Z_{g r}} e^{\frac{\sum_{i \geq j}(i, j)_{n}^{m} \mu_{i j}+n \mu-F_{n}^{m}}{k_{B} T}} .
$$

Comparing Eqs. (14) and (6), it can be noticed that, by using the semi-quantum mechanical approach, $E_{n}{ }^{m}$ is changed to $F_{n}{ }^{m}$.

\section{Ensemble averages}

The ensemble averages for energy $(\langle E\rangle)$, entropy (S), and composition $\left(\left\langle N_{B O}\right\rangle\right)$ are related to the grand partition function by

$$
\langle E\rangle=-k_{B} T \ln Z_{g r}+T S+\langle N\rangle \mu .
$$

The entropy of the liquid is split into configurational and vibrational contributions given by

$S=-k_{B} \sum_{n, m} P_{n}^{m} \ln P_{n}^{m}+\sum_{n, m} P_{n}^{m} S_{n}^{m}=S_{\text {conf }}+S_{\text {vib }}$.

The derivations for Eqs. (15) and (16) are presented in Subsection 3 of the Appendix. The vibrational energy and entropy of a $S_{n}{ }^{m}$ microstate are given by ${ }^{15}$

$$
\begin{gathered}
E_{n}^{m}=\sum_{\nu=1}^{N_{n}^{m}} \hbar \omega_{n}^{m}(v)\left[\frac{1}{2}+\frac{1}{e^{\frac{\hbar \omega_{n}^{m}(v)}{k_{B} T}}-1}\right], \\
S_{n}^{m}=\frac{E_{n}^{m}-F_{n}^{m}}{T} .
\end{gathered}
$$

And, the chemical composition of the glass (from Eq. (3)) is given by

$$
\begin{gathered}
\mathrm{R}_{2} \mathrm{O} \text { or } \mathrm{RO}(\%)=\frac{\langle x\rangle}{1+\langle x\rangle}=\frac{4-\left\langle N_{B O}\right\rangle}{6-\left\langle N_{B O}\right\rangle}, \\
\mathrm{SiO}_{2}(\%)=\frac{1}{1+\langle x\rangle}=\frac{2}{6-\left\langle N_{B O}\right\rangle} .
\end{gathered}
$$

\section{DISCUSSION}

\section{A. Generalization of the model}

The current model describes the probability distribution of silicate units in a binary alkali or alkaline earth silicate glasses where each microstate assumes a single structural configuration. However, the model can be further extended to take into account all structural configurations by labeling a microstate as $S_{n}{ }^{m}(\boldsymbol{\Phi}, \boldsymbol{\Omega})$, where $\boldsymbol{\Phi}$ accounts for the complete internal structure of the silicate unit, encompassing all the vectors from $\varphi_{1}$ to $\varphi_{4} \in \mathbb{R}^{3}$ as shown in Fig. 1 . While $\boldsymbol{\Omega}$ takes into account how the neighboring units are connected to a given unit, encompassing all the vectors from $\omega_{1}$ to $\omega_{4} \in \mathbb{R}^{3}$ (Fig. 1). Together, $\boldsymbol{\Phi}$ and $\boldsymbol{\Omega}$ consider all variations in the bond lengths and bond angles that are associated to a given silicate unit, acknowledging all possible structural configurations. Though, $n$ and $m$ have a discrete probability distribution, $\boldsymbol{\Phi}$ and $\boldsymbol{\Omega}$ could assume a continuous probability distribution. In this case, Equations (1)-(3) change to

$$
\begin{gathered}
\iint_{\boldsymbol{\Phi}, \boldsymbol{\Omega}} \sum_{n, m} P_{n}^{m}(\boldsymbol{\Phi}, \boldsymbol{\Omega}) d \boldsymbol{\Phi} d \boldsymbol{\Omega}=1, \\
\iint_{\boldsymbol{\Phi}, \boldsymbol{\Omega}} \sum_{n, m} E_{n}^{m}(\boldsymbol{\Phi}, \boldsymbol{\Omega}) P_{n}^{m}(\boldsymbol{\Phi}, \boldsymbol{\Omega}) d \boldsymbol{\Phi} d \boldsymbol{\Omega}=\langle E\rangle, \\
\iint_{\boldsymbol{\Phi}, \boldsymbol{\Omega}} \sum_{n, m} n P_{n}^{m}(\boldsymbol{\Phi}, \boldsymbol{\Omega}) d \boldsymbol{\Phi} d \boldsymbol{\Omega}=\left\langle N_{B O}\right\rangle .
\end{gathered}
$$

In the model derived in Section II, $\boldsymbol{\Phi}=\boldsymbol{\Phi}_{T}$, where $\boldsymbol{\Phi}_{T}$ is the associated vector to a silicate tetrahedron, and $\boldsymbol{\Omega}$ would assume some expected value with some variance. Then, integrating $P_{n}{ }^{m}\left(\boldsymbol{\Phi}_{\boldsymbol{T}}, \boldsymbol{\Omega}\right)$ over the entire space of $\boldsymbol{\Omega}$ would yield the value for $P_{n}{ }^{m}$ as shown in the following:

$$
P_{n}=\sum_{m} P_{n}^{m}=\sum_{m} \int_{\boldsymbol{\Phi}, \boldsymbol{\Omega}} P_{n}^{m}\left(\boldsymbol{\Phi}_{\boldsymbol{T}}, \boldsymbol{\Omega}\right) d \boldsymbol{\Phi} d \boldsymbol{\Omega} .
$$

It is also possible that $\boldsymbol{\Phi}$ and $\boldsymbol{\Omega}$ take discrete values in the case when structural units are confined to local minima. Consequently, the integrals over $\boldsymbol{\Phi}$ and $\boldsymbol{\Omega}$ (Eqs. (19)-(21)) would be replaced with summation over all the states of local minima. When multi-component silicate liquid compositions are used, if the added components are network formers (e.g., $\mathrm{Al}_{2} \mathrm{O}_{3}$ or $\mathrm{B}_{2} \mathrm{O}_{3}$ added to silicates), then they could be modelled as additional network units. If units are considered to be atoms of different kind, then one can ignore the internal structure of the unit by dropping off $n$ and $\boldsymbol{\Phi}$. In this case, the model could be applicable to metallic glasses. For other oxide glasses such as borate and phosphate systems, similarly, appropriate internal structures and external correlations should be chosen.

\section{B. LLPS and crystallization}

The introduction of $S_{n}{ }^{m}$ (or $Q_{n}{ }^{i j \ldots}$ ) notation as opposed to previous $Q_{n}$ notation is essential for answering questions concerning LLPS and crystallization. Because of this new notation, which takes into consideration the type of units 
that surround a given unit, the mixing of different units is automatically considered. Consequently, by obtaining probability distribution of $S_{n}{ }^{m}$ units in a given composition by the current model, the amount of LLPS could be calculated. This idea has been experimentally tested using the double quantum $(D Q) N M R$ spectroscopy technique, where the probability distribution of $Q_{n}{ }^{i j \ldots}$ units was measured and the amount of $L L P S$ was estimated. ${ }^{11,16}$

Concerning crystallization, if a particular set of units, which corresponds to a set of points in the $n m$-plane (Fig. 4(a)), undergoes crystallization, then the probability distribution $P_{n}{ }^{m}(\Phi, \Omega)$, for each $S_{n}{ }^{m}(\Phi, \Omega)$ microstate in the $\Phi \Omega$-space, will be sharply peaked, and given by Dirac delta function as

$$
P_{n}^{m}(\Phi, \Omega)=\delta\left(\Phi-\Phi^{\prime}\right) \delta\left(\Omega-\Omega^{\prime}\right),
$$

where $\Phi^{\prime}$ and $\Omega^{\prime}$ are constants corresponding to a particular crystal structure. Therefore, crystallization (or crystal nucleation) of a particular set of $S_{n}{ }^{m}$ units in a supercooled liquid corresponds to a collection of $S_{n}{ }^{m}$ units and sharpening of the $P_{n}{ }^{m}(\Phi, \Omega)$ peak in the $\Phi \Omega$-space.

In the glass forming liquids, the time scales required to access the crystalline states are large. Therefore, these states can be eliminated by assuming some broad distribution of probabilities in the $\Phi \Omega$-space for a given $S_{n}{ }^{m}$ unit. This subject of LLPS and crystallization within the framework of the current model will be expounded in a subsequent paper.

\section{Structural relaxation}

In the last two decades, huge advances have been made in the understanding of the nature of glass and structural relaxation using the potential energy landscape (PEL) approach. ${ }^{17-20}$ The PEL approach uses a canonical ensemble of various structural configurations of large number of atoms. Our present model is fundamentally different; it employs a grand canonical ensemble of structural units that build the glass network and exchange network modifiers and energy from the reservoir. However, the problem of relaxation can be addressed in a similar way as in the PEL approach using the concept of continuously broken ergodicity $(C B E)$ as proposed by Mauro et al. ${ }^{21}$ Here, we consider conditional probabilities $f_{\mathrm{I}, \mathrm{J}}(t)$, which correspond to a system occupying a microstate $J$ after starting in a known state $I$ with subsequent evolution of time $t$, accounting for the actual transition rates between different states. The conditional probabilities would satisfy the following:

$$
\sum_{J} f_{I, J}(t)=1
$$

where $I$ and $J$ are different $S_{n}{ }^{m}(\Phi, \Omega)$ microstates. In the limit of zero and infinite time evolution, the conditional probabilities reduce to the Kronecker delta function $\left(\delta_{I, J}\right)$ and the equilibrium probabilities, respectively, given by

$$
\begin{gathered}
\lim _{t \rightarrow 0} f_{I, J}(t)=\delta_{I, J}, \\
\lim _{t \rightarrow \infty} f_{I, J}(t)=P_{J} .
\end{gathered}
$$

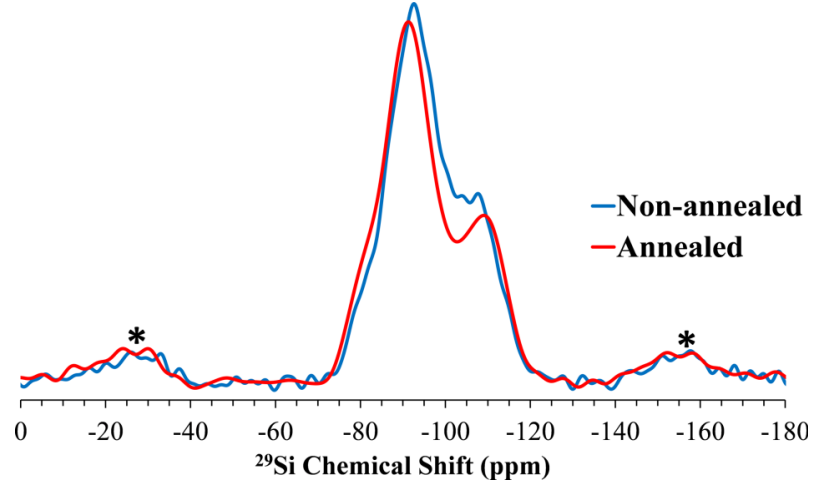

FIG. 2. $N M R$ spectra of annealed and non-annealed (as quenched) $28 \mathrm{Li}_{2} \mathrm{O}-72 \mathrm{SiO}_{2}$ glass, showing structural relaxation. Asterisks indicate spinning side bands.

The conditional entropy is given by

$$
S_{J}(t)=-k_{B} \sum_{J} f_{I, J}(t) \ln f_{I, J}(t) .
$$

The time evolution of the expected value of the configurational is calculated by

$$
S(t)=\sum_{I} P_{I} S_{J}(t)
$$

The time dependent conditional probabilities $f_{\mathrm{I}, \mathrm{J}}(t)$ can be obtained by solving hierarchical master equations given by:

$$
\begin{aligned}
\frac{d f_{I, J}(t)}{d t}= & \sum_{K \neq J} W_{K \rightarrow J}(T(t)) f_{I, K}(t) \\
& -\sum_{K \neq J} W_{J \rightarrow K}(T(t)) f_{I, J}(t),
\end{aligned}
$$

where $W_{K \rightarrow J}$ and $W_{J \rightarrow K}$ are the associated reaction rate constants. After a time evolution $t$, the probability of the state $J$ is given by

$$
P_{J}(t)=\sum_{I} P_{I} f_{I, J}(t)
$$

The relaxation takes place over the entire phase space $\boldsymbol{\Gamma}_{\boldsymbol{s}}$ subjected to available thermal energy and observational time $\left(\tau_{o b s}\right)$. Here we report structural relaxation in a lithium silicate glass from the perspective of the current model using $N M R$ spectroscopy. Figure 2 shows $1 D-N M R$ spectra of a binary lithium silicate glass of composition $28 \% \mathrm{Li}_{2} \mathrm{O}-72 \% \mathrm{SiO}_{2}$ (in moles). One spectrum was recorded on the glass directly quenched from the melt and the other was recorded on the glass quenched and then annealed at $460{ }^{\circ} \mathrm{C}$ for $75 \mathrm{~h}$. The two spectra show clear differences indicating the structural relaxation. The details of the experimental procedure can be found in Subsection 4 of the Appendix.

\section{Test of the model}

In this section, we show how the proposed model can be used in studying silicate based glasses (or liquids) in conjunction with $N M R$ spectroscopy by using an example. The purpose of this section is for the illustration of the usefulness and applicability of the current model. 


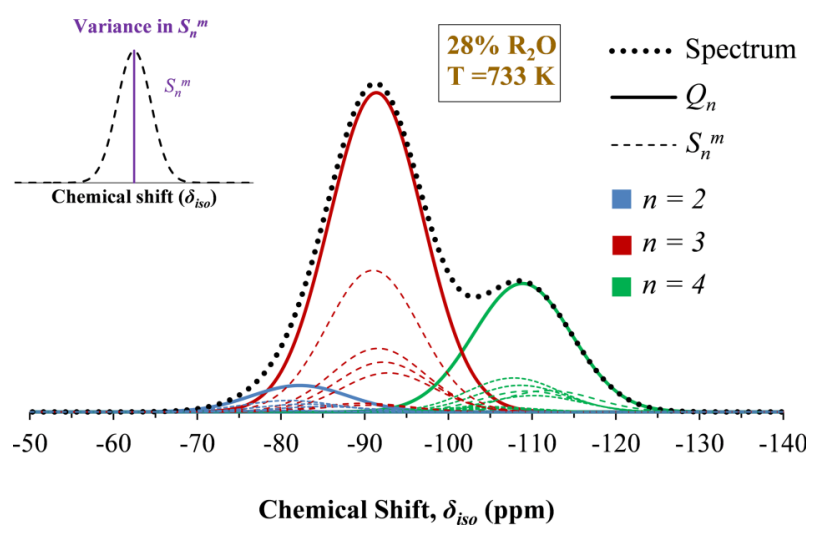

FIG. 3. Simulated $N M R$ spectrum of a hypothetical system using the current model.

The chemical shielding on a particular ${ }^{29} \mathrm{Si}$ nucleus depends on the chemical environment around that nucleus. Therefore, the ${ }^{29} \mathrm{Si}$ isotropic chemical shift $\left(\delta_{i s o}\right)$ of nucleus would be function of all the structural parameters $n, m, \boldsymbol{\Phi}$ and $\boldsymbol{\Omega}: \delta_{i s o}(n, m, \boldsymbol{\Phi}, \boldsymbol{\Omega})$. Since $\boldsymbol{\Phi}$ and $\boldsymbol{\Omega}$ have variance with some expected value, $\delta_{i s o}$ also would have corresponding variance $\left(\sigma^{2}\right)$ and an expected value, $\left\langle\delta_{i s o}\right\rangle$. The variance is given by $^{22}$

$$
\sigma(n, m)^{2}=\left\langle\delta_{i s o}(n, m)\right\rangle^{2}-\left\langle\delta_{i s o}(n, m)\right\rangle^{2} .
$$

We can assume that the variation in $\delta_{i s o}$ for a given $S_{n}{ }^{m}$ unit approximated to a normal distribution (Fig. 3, variance in $\boldsymbol{S}_{\boldsymbol{n}}{ }^{\boldsymbol{m}}$ ). This would be a component of the spectrum associated to a particular $S_{n}{ }^{m}$ unit; and the spectrum of the whole sample, a sum of individual components (Eq. (32)), is shown in Fig. 3. This spectrum corresponds to a hypothetical composition with $28 \% \mathrm{R}_{2} \mathrm{O}$ and is generated by calculating the probabilities $P_{n}{ }^{m}$ in Eq. (14) by assuming some realistic values of $F_{n}{ }^{m}, \delta_{i s o}(n, m)$, and $\sigma(n, m)$ (the procedure is presented in Subsection 5 of the Appendix). Then, the intensity $I\left(\delta_{i s o}\right)$ of the $N M R$ spectrum is given by

$$
I\left(\delta_{i s o}\right) \propto \sum_{n, m} \frac{P_{n}^{m}}{\sigma(n, m) \sqrt{2 \pi}} e^{-\frac{\left(\delta_{i s o}-\left\langle\delta_{i s o}(n, m)\right\rangle\right)^{2}}{2 \sigma(n, m)^{2}}} .
$$

This way, using the current model, NMR spectrum of a given sample could be theoretically computed. Further,

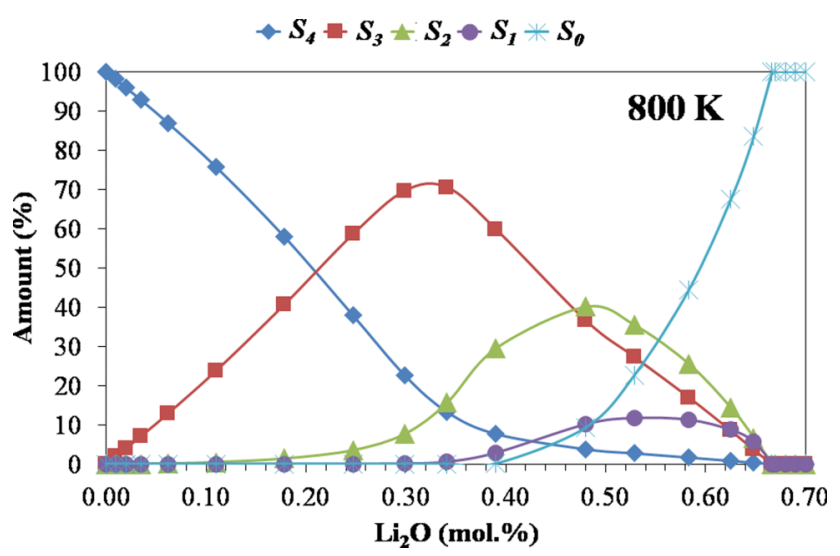

FIG. 5. Variation of probability distribution with composition at $800 \mathrm{~K}$. Dots represent the simulated data points and the lines are just connecting the points to guide the eyes.

using the probability distribution, properties of the liquids can be computed. The variation of properties with temperature for specific heat, entropy, and molar volume is presented in the supplementary material. In order to show relaxation behavior of silicate units of this hypothetical composition, we used a relatively simple concept called broken ergodicity $(B E)$ proposed by Palmer $^{23}$ as opposed to $C B E$ discussed in Sec. III C. In $B E$, we divide the phase space $\boldsymbol{\Gamma}_{\boldsymbol{s}}$ into set of non-ergodic disjoint components where within each component internal ergodicity still exists. In this present example, we divided the phase space ( $\mathrm{nm}$-plane, Fig. 4(a)) into three components: (a) $\boldsymbol{\Gamma}_{\mathbf{1}}=\left\{S_{4}{ }^{1}\right\}$, (b) $\boldsymbol{\Gamma}_{\mathbf{2}}=\left\{S_{4}{ }^{2}, S_{3}{ }^{1}\right\}$, and (c) $\boldsymbol{\Gamma}_{\mathbf{3}}=\boldsymbol{\Gamma}_{\boldsymbol{s}} \cap\left\{S_{4}{ }^{1}, S_{4}{ }^{2}, S_{3}{ }^{1}\right\}$. The reason for selecting these components is because the structural units belonging to $\boldsymbol{\Gamma}_{\mathbf{1}}$ and $\boldsymbol{\Gamma}_{2}$ exist in highly polymerized network; therefore they would not have sufficient time to maintain the ergodicity during the fast quenching of the melt. By enforcing $B E$, probability distribution at some observational time $\left(\tau_{o b s}\right)$ is obtained (Fig. 4(a)). The NMR spectra in Fig. 4(b) are generated by

1. Probability distribution at high temperature $(1600 \mathrm{~K})$ was obtained (which corresponds to $t=0$ ).

2. Then under the $B E$ condition, new probability distribution at $775 \mathrm{~K}$ was obtained (which corresponds to $t=\tau_{o b s}$ ).
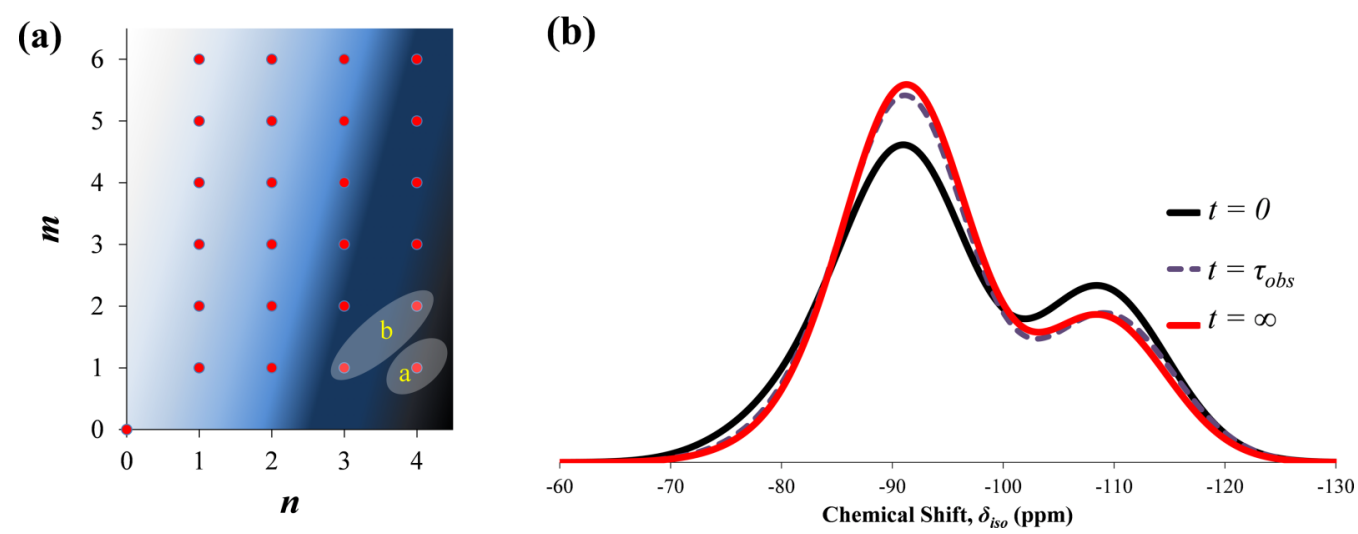

FIG. 4. (a) Phase space in $n$ and $m$ showing the gradient of polymerization: decreasing from dark to light. (b) Relaxation of silicate structural units with time. 
3. Probability distribution without $B E$ condition would yield equilibrium probability at $775 \mathrm{~K}$ (corresponds to $t=\infty$ ).

The relaxation behavior simulated in Fig. 4(b) shows characteristics similar to the experimental observations shown in Fig. 2. Therefore, the as quenched glass without annealing contains a lot of memory effects which can be probed by NMR spectroscopy. This behavior needs to be evaluated for multiple compositions in future studies. Using the same vibrational frequencies, the variation of probability distribution with composition is plotted in Fig. 5.

\section{CONCLUSIONS}

We have presented in this paper a new model based on statistical mechanics to describe the distribution of various silicate units in glasses. We considered the system to be a grand canonical ensemble of silicate units which exchange energy and network modifiers with the reservoir. Several applications where the current model could find its usefulness are mentioned. These include LLPS, crystal nucleation, and glass relaxation. Since statistical mechanics uses microscopic properties to obtain macroscopic properties, several bulk properties of the glass can be easily calculated using the current model.

\section{SUPPLEMENTARY MATERIAL}

See supplementary material for vibrational frequencies used in the simulations and corresponding probability distributions obtained at different temperatures. It also contains plots illustrating the effect of network speciation on several properties of the glass.

\section{ACKNOWLEDGMENTS}

This work was developed within the scope of the project CICECO-Aveiro Institute of Materials, No. POCI-01-0145FEDER-007679 (FCT Reference No. UID/CTM/50011/2013), financed by national funds through the FCT/MEC and when appropriate co-financed by FEDER under the PT2020 and partially supported by the JECS trust (Contract No. 201478). Anuraag Gaddam is grateful for financial support from CICECO.

L.M. thanks the Chevreul Institute (FR 2638) for providing access to NMR measurements. Chevreul Institute is supported by the "Ministère de l'Enseignement Supérieur et de la Recherche", the "Région Nord-Pas de calais" and the "Fonds Européen de Développement des Régions".

\section{APPENDIX: DERIVATION OF NETWORK CONSTRAINTS, PROBABILITY DISTRIBUTION AND ENTROPY, AND EXPERIMENTAL PROCEDURE}

\section{Network connectivity constraints}

In a given glass composition, $\mathrm{BO}$ s characterized by $O_{i j}$ must be conserved. Therefore Equations (A1)-(A10) corresponding to 10 different $O_{i j}$ oxygens must hold, where $p_{4}{ }^{i j k l}, p_{3}{ }^{i j k}, p_{2}{ }^{i j}$, and $p_{1}{ }^{i}$ are the probabilities (notice lower case " $p$ " as opposed to upper case " $P$ " in $S$ notation) associated with $Q_{4}{ }^{i j k l}, Q_{3}{ }^{i j k}, Q_{2}{ }^{i j}$, and $Q_{1}{ }^{i}$ units. The $Q$ notation is employed here because it is easier to see the connection between right and left hand sides of the equations,

$$
\begin{gathered}
\boldsymbol{O}_{\mathbf{1 1}}: p_{1}^{1}=p_{1}^{1}, \\
\boldsymbol{O}_{\mathbf{1 2}}: p_{1}^{2}=\sum_{j \neq 1} p_{2}^{1 j}+2 p_{2}^{11},
\end{gathered}
$$

$$
\boldsymbol{O}_{13}: p_{1}^{3}=\sum_{j, k \neq 1} p_{3}^{1 j k}+2 \sum_{k \neq 1} p_{3}^{11 k}+3 p_{3}^{111} \text {, }
$$

$$
\boldsymbol{O}_{\mathbf{1 4}}: p_{1}^{4}=\sum_{j, k, l \neq 1} p_{4}^{1 j k l}+2 \sum_{k, l \neq 1} p_{4}^{11 k l}+3 \sum_{l \neq 1} p_{4}^{111 l}+4 p_{4}^{1111} \text {, }
$$$$
\boldsymbol{O}_{\mathbf{2 2}}: \sum_{j \neq 2} p_{2}^{2 j}+2 p_{2}^{22}=\sum_{j \neq 2} p_{2}^{2 j}+2 p_{2}^{22},
$$

$\boldsymbol{O}_{\mathbf{2 3}}: \sum_{j \neq 3} p_{2}^{3 j}+2 p_{2}^{33}=\sum_{j, k \neq 2} p_{3}^{2 j k}+2 \sum_{k \neq 2} p_{3}^{22 k}+3 p_{3}^{222}$,

$$
\begin{aligned}
\boldsymbol{O}_{\mathbf{2 4}}: \sum_{j \neq 4} p_{2}^{4 j}+2 p_{2}^{44}= & \sum_{j, k, l \neq 2} p_{4}^{2 j k l}+2 \sum_{k, l \neq 2} p_{4}^{22 k l} \\
& +3 \sum_{l \neq 2} p_{4}^{222 l}+4 p_{4}^{2222}
\end{aligned}
$$

$$
\begin{gathered}
\boldsymbol{O}_{\mathbf{3 3}}: \sum_{j, k \neq 3} p_{3}^{3 j k}+2 \sum_{k \neq 3} p_{3}^{33 k}+3 p_{3}^{333} \\
=\sum_{j, k \neq 3} p_{3}^{3 j k}+2 \sum_{k \neq 3} p_{3}^{33 k}+3 p_{3}^{333}, \\
\boldsymbol{O}_{\mathbf{3 4}}: \sum_{j, k \neq 4} p_{3}^{4 j k}+2 \sum_{k \neq 4}^{44 k} p_{3}^{44 k}+3 p_{3}^{444} \\
=\sum_{j, k, l \neq 4} p_{4}^{3 j k l}+2 \sum_{k, l \neq 4} p_{4}^{33 k l}+3 \sum_{l \neq 4} p_{4}^{333 l}+4 p_{4}^{3333}, \\
\boldsymbol{O}_{\mathbf{4 4}}: \sum_{j, k, l \neq 4} p_{4}^{4 j k l}+2 \sum_{k, l \neq 4} p_{4}^{44 k l}+3 \sum_{l \neq 4} p_{4}^{444 l}+4 p_{4}^{4444} \\
=\sum_{j, k, l \neq 4} p_{4}^{4 j k l}+2 \sum_{k, l \neq 4} p_{4}^{44 k l}+3 \sum_{l \neq 4} p_{4}^{444 l}+4 p_{4}^{4444} .
\end{gathered}
$$

Equations (A1)-(A10) can be represented as follows:

$$
\sum_{n, m}(i, j)_{n}^{m} P_{n}^{m}=k_{i j}
$$

The coefficients $(i, j)_{n}{ }^{m}$ are constants associated to each equation representing a given $O_{i j}$ BO. Further, according to (A1)-(A10), the values of $(i, j)_{n}{ }^{m}=0 \forall i=j$ and $k_{i j}=0 \forall i, j$. The values of the constants are presented in Table I.

\section{Derivation for the probabilities $P_{n}{ }^{m}$}

The solution given by Eq. (6) is obtained from the Lagrange function $\mathcal{L}\left(P_{n}{ }^{m}\right)$ with the Lagrange multipliers $\alpha$, $\beta$ and $\gamma$ given by 


$$
\begin{aligned}
\mathcal{L}\left(P_{n}^{m}\right)= & k_{B} \sum_{n, m}\left(P_{n}^{m} \ln P_{n}^{m}\right)+\alpha\left[\sum_{n, m} P_{n}^{m}-1\right] \\
& +\beta\left[\sum_{n, m} E_{n}^{m} P_{n}^{m}-\langle E\rangle\right]+\gamma\left[\sum_{n, m} n P_{n}^{m}-2\left\langle N_{B O}\right\rangle\right] \\
& +\sum_{i, j} \gamma_{i j}\left[\sum_{n, m}(i, j)_{n}^{m} P_{n}^{m}-k_{i j}\right] .
\end{aligned}
$$

Differentiating $\mathcal{L}\left(P_{n}{ }^{m}\right)$ with respect to $P_{n}{ }^{m}$ would equal zero,

$$
\begin{aligned}
\frac{\partial \mathcal{L}\left(P_{n}^{m}\right)}{\partial P_{n}^{m}}= & k_{B}\left(1+\ln P_{n}^{m}\right)+\alpha+\beta E_{n}^{m} \\
& +\gamma n+\sum_{i, j} \gamma_{i j}(i, j)_{n}^{m}=0 .
\end{aligned}
$$

Rearranging,

$\ln P_{n}^{m}=-\ln Z_{g r}-\frac{\beta E_{n}^{m}}{k_{B}}-\frac{n \gamma}{k_{B}}-\frac{\sum_{i, j}(i, j)_{n}^{m} \gamma_{i j}}{k_{B}}$,

where $\ln Z_{g r}=\frac{\left(\alpha+k_{B}\right)}{k_{B}}$ and substituting Eq. (A13) in Eq. (6)

$$
\begin{aligned}
S= & -k_{B} \sum_{n, m}\left(-P_{n}^{m} \ln Z_{g r}-P_{n}^{m} \frac{\beta E_{n}^{m}}{k_{B}}-P_{n}^{m} \frac{n \gamma}{k_{B}}\right. \\
& \left.-P_{n}^{m} \frac{\sum_{i, j} \gamma_{i j}(i, j)_{n}^{m}}{k_{B}}\right) .
\end{aligned}
$$

Solving the above equation using Equations (1)-(4) gives

$$
S=k_{B} \ln Z_{g r}+\beta\langle E\rangle+2 \gamma\left\langle N_{B O}\right\rangle+\sum_{i, j} \gamma_{i j} k_{i j} .
$$

Rearranging,

$$
\langle E\rangle=\frac{1}{\beta} S-\frac{k_{B}}{\beta} \ln Z_{g r}-\frac{\gamma}{\beta}\left(2\left\langle N_{B O}\right\rangle\right)-\sum_{i, j} \frac{\gamma_{i j}}{\beta} k_{i j} .
$$

Differentiating,

$$
d\langle E\rangle=\frac{1}{\beta} d S-\frac{k_{B}}{\beta} d \ln Z_{g r}-\frac{\gamma}{\beta} d\left(2\left\langle N_{B O}\right\rangle\right)-\sum_{i, j} \frac{\gamma_{i j}}{\beta} d k_{i j} .
$$

Comparing the above equation with the fundamental thermodynamic relation ${ }^{24}$

$$
d E=T d S-P d V+\sum \mu_{i} d n_{i}
$$

would yield

$$
\begin{gathered}
\beta=\frac{1}{T}, \\
\gamma=-\frac{\mu}{T}, \\
\gamma_{i j}=-\frac{\mu_{i j}}{T} .
\end{gathered}
$$

Therefore, substituting Eqs. (A15)-(A17) into Eq. (A13) and rearranging gives

$$
P_{n}^{m}=\frac{1}{Z_{g r}} e^{\frac{\sum_{i, j}(i, j)_{n}^{m} \mu_{i j}+n \mu-E_{n}^{m}}{k_{B} T}}
$$

\section{Entropy of the liquid}

The entropy of the liquid is given by

$$
\begin{aligned}
S= & -k_{B} \sum_{n, m, \boldsymbol{k}} P_{n}^{m}(\boldsymbol{k}) \ln P_{n}^{m}(\boldsymbol{k}), \\
S= & -k_{B} \sum_{n, m, \boldsymbol{k}} P_{n}^{m}(\boldsymbol{k})\left[-\ln Z_{g r}+\frac{\sum_{i \geq j}(i, j)_{n}^{m} \mu_{i j}}{k_{B} T}+\frac{n \mu}{k_{B} T}\right. \\
& \left.-\sum_{v=1}^{N_{n}^{m}}\left(\frac{1}{2}+k(v)\right) \frac{\hbar \omega_{n}^{m}(v)}{k_{B} T}\right], \\
S= & k_{B} \ln Z_{g r}-\frac{\left\langle N_{B O}\right\rangle \mu}{T}+\sum_{n, m, \boldsymbol{k}} P_{n}^{m}(\boldsymbol{k}) \sum_{v=1}^{N_{n}^{m}}\left(\frac{1}{2}+k(v)\right) \\
& \times \frac{\hbar \omega_{n}^{m}(v)}{T},
\end{aligned}
$$$$
S=k_{B} \ln Z_{g r}-\frac{\left\langle N_{B O}\right\rangle \mu}{T}+\sum_{n, m} P_{n}^{m} \sum_{v=1}^{N_{n}^{m}} \frac{\hbar \omega_{n}^{m}(v)}{T}
$$$$
\times\left[\frac{1}{2}+\frac{1}{e^{\frac{\hbar \omega_{n}^{m}(\nu)}{k_{B} T}}-1}\right],
$$$$
S=k_{B} \ln Z_{g r}-\frac{\left\langle N_{B O}\right\rangle \mu}{T}+\sum_{n, m} \frac{P_{n}^{m} E_{n}^{m}}{T},
$$$$
S=k_{B} \ln Z_{g r}-\frac{N_{B O} \mu}{T}+\frac{E}{T} .
$$

Further, the entropy can be split into configurational and vibrational parts

$$
\begin{aligned}
S= & k_{B} \ln Z_{g r}-\frac{\left\langle N_{B O}\right\rangle \mu}{T}+\sum_{n, m} \frac{P_{n}^{m} E_{n}^{m}}{T}, \\
S= & k_{B} \ln Z_{g r}-\frac{\left\langle N_{B O}\right\rangle \mu}{T}+\sum_{n, m} \frac{P_{n}^{m}\left(F_{n}^{m}+T S_{n}^{m}\right)}{T}, \\
S= & \sum_{n, m} P_{n}^{m}\left[k_{B} \ln Z_{g r}-\frac{\sum_{i \geq j}(i, j)_{n}^{m} \mu_{i j}}{T}-\frac{n \mu}{T}+\frac{F_{n}^{m}}{T}+S_{n}^{m}\right], \\
S= & -k_{B} \sum_{n, m} P_{n}^{m}\left[\ln Z_{g r}+\frac{\sum_{i \geq j}(i, j)_{n}^{m} \mu_{i j}}{k_{B} T}+\frac{n \mu}{k_{B} T}-\frac{F_{n}^{m}}{k_{B} T}\right] \\
& +\sum_{n, m} P_{n}^{m} S_{n}^{m}, \\
S= & -k_{B} \sum_{n, m} P_{n}^{m} \ln P_{n}^{m}+\sum_{n, m} P_{n}^{m} S_{n}^{m}, \\
S= & S_{\text {conf }}+S_{v i b} .
\end{aligned}
$$

\section{Experimental procedure}

For the preparation of the glass, $\mathrm{SiO}_{2}$ and $\mathrm{Li}_{2} \mathrm{CO}_{3}$ with purity $>99 \%$ were weighed in required amounts, mixed by ball milling, and then calcined at $800{ }^{\circ} \mathrm{C}$ in alumina crucibles for $1 \mathrm{~h}$ in air. The calcined powder was crushed in a mortar and transferred to a Pt crucible for melting at a temperature of $1550{ }^{\circ} \mathrm{C}$ for $1 \mathrm{~h}$ in air. Bulk (monolithic) bar shaped glasses were prepared by pouring the melt on a bronze mold. One sample was annealed at $460{ }^{\circ} \mathrm{C}$ for $75 \mathrm{~h}$. X-ray diffraction analysis (not shown) confirmed that the samples were fully amorphous. 
${ }^{29}$ Si MAS-NMR spectra were recorded on both annealed and non-annealed glass samples crushed into fine powders. The NMR spectrometer (BRUKER Avance III) was operated at a Larmor frequency of $79.5 \mathrm{MHz}$ with a $9.4 \mathrm{~T}$ magnetic field, using a $7 \mathrm{~mm}$ rotor rotating at $5 \mathrm{kHz}$. The samples were excited with a $90^{\circ}$ flip angle using $900 \mathrm{~s}$ delay time. Both spectra were obtained after Fourier transformation of 64 scans of free induction decays (FID). Tetramethylsilane was used as chemical shift reference at $0 \mathrm{ppm}$.

\section{Details of simulation}

The $N M R$ spectrum obtained from the annealed glass was deconvoluted using DMfit software ${ }^{25}$ for the units $Q_{2}$, $Q_{3}$, and $Q_{4}$ using mixed Gaussian/Lorentzian line shapes. The amounts of the units obtained were $6 \%, 66 \%$, and $28 \%$ for the units $Q_{2}, Q_{3}$, and $Q_{4}$, respectively. Using the current model, the $S_{n}{ }^{m}$ distribution was simulated by fitting the appropriate $\omega_{n}{ }^{m}$ values in order to simulate a realistic probability distribution that is in agreement with the experimentally measured distribution. The fitted $\omega_{n}{ }^{m}$ values and the probability distributions are presented in Tables S1 and S2, respectively.

${ }^{1}$ W. H. Zachariasen, J. Am. Chem. Soc. 54, 3841 (1932).

${ }^{2}$ J. F. Stebbins and S. Sen, J. Non. Cryst. Solids 368, 17 (2013).

${ }^{3}$ H. W. Nesbitt, G. M. Bancroft, G. S. Henderson, R. Ho, K. N. Dalby, Y. Huang, and Z. Yan, J. Non. Cryst. Solids 357, 170 (2011).

${ }^{4}$ R. Dupree, N. Ford, and D. Holland, Phys. Chem. Glasses 28, 78 (1987).
${ }^{5}$ M. E. Brandriss and J. F. Stebbins, Geochim. Cosmochim. Acta 52, 2659 (1988).

${ }^{6}$ B. A. Shakhmatkin and N. M. Vedishcheva, J. Non. Cryst. Solids 171, 1 (1994).

${ }^{7}$ B. A. Shakhmatkin, N. M. Vedishcheva, M. M. Shultz, and A. C. Wright, J. Non. Cryst. Solids 177, 249 (1994).

${ }^{8}$ N. M. Vedishcheva, B. A. Shakhmatkin, M. M. Shultz, and A. C. Wright, J. Non. Cryst. Solids 196, 239 (1996).

${ }^{9}$ J. Schneider, V. R. Mastelaro, E. D. Zanotto, B. A. Shakhmatkin, N. M. Vedishcheva, A. C. Wright, and H. Panepucci, J. Non. Cryst. Solids 325, 164 (2003).

${ }^{10}$ J. C. Mauro, J. Chem. Phys. 138, 12A522 (2013).

${ }^{11}$ L. Olivier, X. Yuan, A. N. Cormack, and C. Jäger, J. Non. Cryst. Solids 293-295, 53 (2001).

${ }^{12}$ J. Machacek, O. Gedeon, and M. Liska, J. Non. Cryst. Solids 352, 2173 (2006).

${ }^{13}$ M. Edén, Annu. Rep., Sect. C: Phys. Chem. 108, 177 (2012).

${ }^{14}$ S. Brawer, Phys. Rev. B 11, 3173 (1975).

${ }^{15}$ Problems in Thermodynamics and Statistical Physics, edited by P. T. Landsberg (Pion Limited, Bristol, 1989).

${ }^{16}$ L. Martel, D. Massiot, and M. Deschamps, J. Non. Cryst. Solids 390, 37 (2014).

${ }^{17}$ P. G. Debenedetti and F. H. Stillinger, Nature 410, 259 (2001).

${ }^{18}$ F. Sciortino, J. Stat. Mech.: Theory Exp. 2005, P05015.

${ }^{19}$ J. C. Mauro and M. M. Smedskjaer, J. Non. Cryst. Solids 396-397, 41 (2014).

${ }^{20}$ P. K. Gupta, J. Non. Cryst. Solids 407, 154 (2015).

${ }^{21}$ J. C. Mauro, P. K. Gupta, and R. J. Loucks, J. Chem. Phys. 126, 184511 (2007).

${ }^{22}$ A. W. Drake, Fundamentals of Applied Probability Theory (McGraw-Hill, New York, 1988).

${ }^{23}$ R. G. Palmer, Adv. Phys. 31, 669 (1982).

${ }^{24} \mathrm{D}$. R. Gaskell, Introduction to the Thermodynamics of Materials, 4th ed. (Taylor \& Francis Group, New York, 2003).

${ }^{25}$ D. Massiot, F. Fayon, M. Capron, I. King, S. Le Calvé, B. Alonso, J.-O. Durand, B. Bujoli, Z. Gan, and G. Hoatson, Magn. Reson. Chem. 40, 70 (2002). 